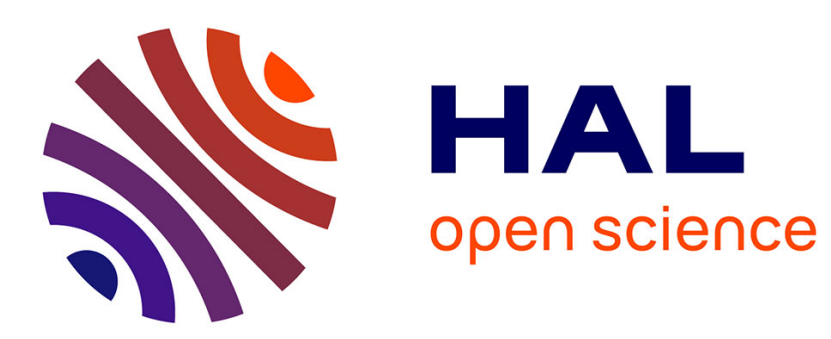

\title{
Dissipation et claquage thermique dans les solides non métalliques soumis à un champ électrique intense.
}

\author{
Rui Coelho, R. Goffaux
}

\section{To cite this version:}

Rui Coelho, R. Goffaux. Dissipation et claquage thermique dans les solides non métalliques soumis à un champ électrique intense.. Revue de Physique Appliquée, 1981, 16 (3), pp.67-75. 10.1051/rphysap:0198100160306700 . jpa-00244903

\section{HAL Id: jpa-00244903 https://hal.science/jpa-00244903}

Submitted on 1 Jan 1981

HAL is a multi-disciplinary open access archive for the deposit and dissemination of scientific research documents, whether they are published or not. The documents may come from teaching and research institutions in France or abroad, or from public or private research centers.
L'archive ouverte pluridisciplinaire HAL, est destinée au dépôt et à la diffusion de documents scientifiques de niveau recherche, publiés ou non, émanant des établissements d'enseignement et de recherche français ou étrangers, des laboratoires publics ou privés. 


\title{
Dissipation et claquage thermique dans les solides non métalliques soumis à un champ électrique intense.
}

\author{
R. Coelho et R. Goffaux $\left({ }^{* *}\right)$ \\ Laboratoire de Physique des Décharges (*), École Supérieure d'Électricité, Plateau du Moulon, 91190 Gif-sur-Yvette, France \\ $\left({ }^{* *}\right)$ Laboratoire de Recherches, Ateliers de Constructions de Charleroi, B.P. no 4, 6000 Charleroi, Belgique
}

(Reçu le 13 octobre 1980, révisé le 9 décembre 1980, accepté le 10 décembre 1980)

\begin{abstract}
Résumé. - Cet article est le premier d'une série consacrée au comportement de matériaux non métalliques soumis à un champ élevé. Dans le travail présenté ci-dessous, on étudie la variation du courant de conduction avec la valeur de la tension continue (ou TBF) appliquée à un échantillon plan, en tenant compte de la dissipation interne. On discute la répartition de la température, du potentiel et du champ ainsi que la forme des caractéristiques couranttension en examinant le rôle d'une éventuelle discontinuité de température aux interfaces. On montre qu'une telle discontinuité entraîne l'existence d'une branche à pente négative sur la caractéristique. On en tire quelques conclusions relatives au claquage thermique. On montre que l'existence des gradients de température dans l'épaisseur de l'échantillon provoque une polarisation par charge d'espace, et on calcule l'incrément de permittivité statique qui en découle. Dans les articles en cours de préparation on étudiera les conditions de formation d'un canal conducteur, et les conséquences de l'accumulation de charge aux interfaces.
\end{abstract}

\begin{abstract}
This paper is the first of a series devoted to the behaviour of non-metallic materials submitted to a high field. In the work presented here, we investigate the variation of the conduction current with the value of the d.c. or v.l.f. voltage applied on a plane sample, taking into account the internal heat dissipation. We discuss the temperature, voltage and field distributions in the sample, as well as the shape of the current-voltage characteristics and the effect on this shape of steps in the temperature profile at the interfaces. We show that if such steps are present, the characteristics contains a zone of negative slope, and we draw from this conclusions relative to the thermal breakdown. Finally we show that the presence of temperature gradients in the electrically stressed samples induces a space-charge polarization, and we derive the static permittivity related to this space-charge. In the articles presently under preparation, we shall deal with the formation of a conducting bridge across the sample and the consequences of the storage of charge at the interfaces.
\end{abstract}

\section{Liste des symboles utilisés.}

$\sigma$ conductivité (à partir de la relation (10), on a c posé $\left.\sigma_{0}=\sigma\left(T_{0}\right)\right)$.

$U$ énergie d'activation.

$T$ température absolue $\left(T_{0}\right.$ : ambiante, $T_{1}$ : aux interfaces, $T_{\mathrm{m}}$ : au plan médian, $\left.t=\left(T_{\mathrm{m}}-T_{1}\right) / \theta\right)$.

$\theta$ constante de température.

$k$ constante de Boltzmann.

E champ électrique.

I densité de courant $i:$ densité de courant réduite).

$V$ tension ( $v:$ tension réduite).

$W$ puissance (par unité de surface). (en indice : relatif aux conditions critiques).

(en indice : relatif à la forme $\sigma(E) \propto E^{n}$ ).

$L$ épaisseur de l'échantillon.

$\Gamma$ coefficient d'échange thermique aux interfaces.

$x \quad \theta / T_{0}$.

$K$ conductivité thermique.

$z \quad$ ordonnée courante.

a épaisseur caractéristique (eq. (41)).

U tension réduite.

J courant réduit.

$\gamma \quad \Gamma L / 4 K$.

moment dipolaire par unité de surface.

permittivité de la matrice.

polarisation.

$\left({ }^{*}\right)$ E.R. 114 du C.N.R.S. 
1. Introduction. - Dans le présent mémoire, on étudie le courant de conduction passant à travers une plaque homogène d'épaisseur $L$ d'un matériau de type semiconducteur soumis à une haute tension continue.

La conductivité d'un tel matériau varie avec la température suivant une loi d'Arrhénius :

$$
\sigma(T)=\sigma_{\infty} \exp (-U / k T) .
$$

Toutefois, pour simplifier les calculs, la relation (1) sera remplacée ici par l'approximation classique [1] :

$$
\sigma(T)=\sigma\left(T_{0}\right) \exp \left(\frac{T-T_{0}}{\theta}\right)
$$

La température $\theta$ de la relation (2) peut s'exprimer en fonction de l'énergie d'activation $U$ et de la température ambiante $T_{0}$ en identifiant les dérivées logarithmiques des deux relations :

$$
\frac{1}{\theta}=\frac{U}{k T^{2}} \text {. }
$$

Pour $U=1 \mathrm{eV}$ et $T_{0}=300 \mathrm{~K}$, la relation (3) donne $\theta=7,5^{\circ}$. Les écarts qu'entraîne l'utilisation de l'approximation (2) par rapport à la relation (3) sont discutés en Annexe I.

Tout en conservant le facteur de température exponentiel de la relation (2), on considérera aussi le cas où la conductivité $\sigma$ dépend également du champ $E[2,3]$ :

$$
\sigma(T, E)=\sigma\left(T_{0}\right) \exp \left(\frac{T-T_{0}}{\theta}\right) f(E)
$$

$f(E)$ étant une fonction croissante de $E$, puisqu'à champ élevé, le courant dans les matériaux semiisolants augmente plus vite que le champ.

Dans la première partie à caractère essentiellement didactique (sections 2 et 3 ), on supposera que l'élévation de température provoquée dans le matériau par la dissipation est uniforme, mais que l'ambiance reste à la température uniforme $T_{0}$, de sorte que le profil de température à l'équilibre, uniforme à l'intérieur et à l'extérieur du matériau, subit à l'interface une discontinuité d'amplitude $\left(T-T_{0}\right)$.

Cette hypothèse simplificatrice est faite dans la plupart des traitements élémentaires du problème. Toutefois, elle implique que la conductivité thermique du matériau, de même que celle de l'ambiance, est infinie, et qu'un transfert de chaleur de type convectif, de la forme $\Gamma\left(T-T_{0}\right)$ se produise à chaque interface entre le matériau et l'ambiance.

En fait, la conservation du flux de chaleur dans un matériau réel implique que le cour (ici le plan médian) est plus chaud que les faces.

Toutefois, la densité de courant $I=\sigma E$ est uniforme dans le matériau à l'équilibre, et par suite la puissance $I E$ dissipée par unité d'épaisseur, proportionnelle à $E$, est moins intense dans le plan médian où $\sigma$ est maximum (car la température y est maximale) qu'au voisinage des faces. La variation de $\sigma$ avec le champ $E$ (4) tend à uniformiser la température et le champ dans l'épaisseur du matériau, et donc à justifier partiellement l'approximation de la température uniforme - au moins pour un échantillon épais.

2. Bilan d'énergie et conditions critiques. - L'argumentation classique permettant de préciser les conditions d'équilibre de l'échantillon isotherme repose sur le bilan d'énergie (Fig. 1). A l'équilibre, la puissance

$$
W=I V=\sigma E^{2} L
$$

dissipée par effet Joule par unité de surface, doit s'échapper par les interfaces. La température d'équilibre $T$ résulte de l'égalité

$$
I V=\sigma(T) E^{2} L=2 \Gamma\left(T-T_{0}\right)
$$

le facteur 2 provenant du fait que l'échange thermique aux deux interfaces est supposé le même.

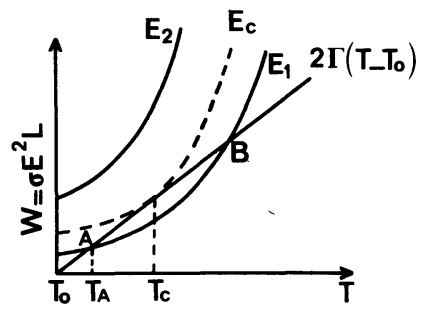

Fig. 1. - Dissipation et transfert d'énergie pour un matériau isotherme en fonction de la température. Les courbes $E$ représentent la dissipation pour différentes valeurs du champ appliqué $E_{1}<E_{\mathrm{c}}<E_{2}$. $E_{\mathrm{c}}$ est le champ critique de claquage thermique. La droite $2 \Gamma\left(T-T_{0}\right)$ représente l'énergie transférée à l'ambiance par les parois. La courbe $E_{1}$ coupe la droite de transfert en deux points $\mathrm{A}$ (qui représente un équilibre stable à la température $T_{\mathrm{A}}$ ) et $\mathrm{B}$ (qui représente un équilibre instable à $T_{\mathrm{B}}$ ).

[Dissipation and energy transfer for an isothermal sample versus temperature. The $E$ curves represent the dissipation for various values of applied field $E_{1}<E_{\mathrm{c}}<E_{2} . E_{\mathrm{c}}$ is the critical field for thermal breakdown. The line $2 \Gamma\left(T-T_{0}\right)$ represents the energy transferred to the ambiance through the interfaces. Curve $E_{1}$ intersects the transfer line in two points $A$ (representing steady state at temperature $T_{\mathrm{A}}$ ) and $\mathrm{B}$ (representing an unstable state at $T_{\mathrm{B}}$ ).]

Dans la figure 1, les courbes représentent la puissance dissipée par unité de surface $\sigma E^{2} L$ pour différentes valeurs de $E$ (courbes affines les unes des autres dans le rapport $E^{2}$ ) et la droite représente la puissance sortante $2 \Gamma\left(T-T_{0}\right)$ par unité de surface.

On voit que pour toute valeur du champ $E$ inférieure à la valeur critique $E_{\mathrm{c}}$, la courbe de dissipation coupe la droite de fuite en deux points. Le point $A$ représente un équilibre stable. En effet, l'échantillon se réchauffe si $T<T_{\mathrm{A}}$, puisque la chaleur entrante excède alors la chaleur sortante, et il se refroidit si $T>T_{\mathrm{A}}$, puisque la chaleur sortante excède alors la chaleur entrante. Quant au point $B$, il représente un équilibre instable inaccessible. 
Pour toute valeur du champ $E$ supérieure à $E_{c}$, la chaleur entrante est toujours supérieure à la chaleur sortante, de sorte qu'aucun équilibre n'est possible. De ceci, il résulte que le champ critique de claquage $E_{\mathrm{c}}$ est celui pour lequel la courbe de puissance entrante est tangente à la droite de puissance sortante.

Les conditions critiques de claquage thermique (champ, courant, température...) sont donc réalisées quand on a à la fois :

$$
\sigma E^{2} L=2 \Gamma\left(T-T_{0}\right)
$$

et

$$
\frac{\mathrm{d}}{\mathrm{d} T}\left(\sigma E^{2} L\right)=2 \Gamma
$$

Si on utilise la loi d'Arrhénius pour la conductivité, on obtient une équation quadratique pour la température critique $T_{\mathrm{c}}$, et les relations donnant la tension et le courant critiques étant relativement lourdes, les calculs analytiques deviennent rapidement inextricables.

L'utilisation pour $\sigma$ de l'approximation de Wagner (2) conduit au contraire à des calculs simples, dont les conséquences relatives aux conditions critiques sont résumées dans le tableau I, colonne 2 . A la colonne 3 du même tableau, on a porté l'écart relatif entre les quantités critiques déduites des relations (1) et (2). La validité de l'approximation de Wagner, étudiée en Annexe I, justifie l'emploi de cette approximation dans les calculs ultérieurs.

Tableau I. - Les conditions critiques et les écarts relatifs, donnés en fonction de $x=\theta / T_{0}$, qui vaut $2,5 \times 10^{-2}$ pour $U=1 \mathrm{eV}$.

[The critical conditions from eq. (2), and the relative errors in terms of $x=\theta / T_{0} .\left(x=2.5 \times 10^{-2}\right)$ for $U=1 \mathrm{eV}$.]

\begin{tabular}{lccc} 
Quantités critiques & \multicolumn{2}{c}{ Formules dérivées de (2) } & Ecart relatif \\
- & \multicolumn{2}{c}{-} & - \\
Température $T_{\mathrm{c}}$ & $T_{\mathrm{o}}+\theta$ & $(8)$ & $2 x^{2}$ \\
Puissance $W_{\mathrm{c}}$ & $2 \Gamma \theta$ & $(9)$ & $2 x$ \\
Potentiel $V_{\mathrm{c}}$ & $\left(2 \Gamma \theta L / \sigma_{0} \mathrm{e}\right)^{1 / 2}$ & $(10)$ & $x / 2$ \\
Champ $E_{\mathrm{c}}$ & $\left(2 \Gamma \theta / L \sigma_{0} \mathrm{e}\right)^{1 / 2}$ & $(11)$ & $x / 2$ \\
Courant $I_{\mathrm{c}}$ & $\left(2 \Gamma \theta \sigma_{0} \mathrm{e} / L\right)^{1 / 2}$ & $(12)$ & $3 x / 2$
\end{tabular}

(Le symbole e dans les relations (10) à (12) représente la base des logarithmes naturels.)

3. La caractéristique courant-tension. - 3.1 ÉCHANTILLON OHMIQUE. - En tenant compte de la relation (8), l'équation d'équilibre (6) s'écrit :

$$
I V=2 \Gamma \theta \ln \left(\sigma / \sigma_{0}\right)
$$

mais comme $I=\sigma V / L, \sigma / \sigma_{0}=L I / \sigma_{0} V$, et (13) devient :

$$
I V=2 \Gamma \theta \ln \left(L I / \sigma_{0} V\right) .
$$

La relation (14) représente l'équation intrinsèque de la caractéristique $I(V)$. On peut l'écrire sous forme non dimensionnelle en utilisant les variables réduites $i=I / I_{\mathrm{c}}$ et $v=V / V_{\mathrm{c}}$ :

$$
i v=\ln \left(\mathrm{e} \frac{i}{v}\right)=1+\ln \frac{i}{v}
$$

Cette équation transcendante ne permet pas d'exprimer $i$ en fonction de $v$. Toutefois, on peut obtenir un développement limité de $i(v)$ au voisinage de l'origine $(i \sim v \sim 0)$ :

$$
i(v)=\frac{v}{\mathrm{e}}\left(1+\frac{v^{2}}{\mathrm{e}}+\cdots\right)
$$

montrant que le courant augmente plus rapidement que la tension appliquée, comme le laisse prévoir l'augmentation de $\sigma$ avec le courant.

Ceci peut être précisé par le calcul direct de la pente $\mathrm{d} i / \mathrm{d} v$ de la caractéristique réduite. Par différentiation de la relation (15) on obtient :

$$
\frac{\mathrm{d} i}{\mathrm{~d} v}=\frac{1+i v}{1-i v} \frac{\mathrm{i}}{v}
$$

et on voit que la pente augmente avec la puissance dissipée jusqu'à devenir infinie pour $i v=1$, c'est-àdire $I V=I_{\mathrm{c}} V_{\mathrm{c}}=2 \Gamma \theta$.

Si la puissance dissipée dépasse $2 \Gamma \theta$, la pente $\mathrm{d} i / \mathrm{d} v$ devient négative, et la caractéristique s'incurve vers l'axe des $i$ (Fig. 2), de sorte que la tension à l'équilibre aux bornes de l'échantillon ne peut en aucun cas dépasser $V_{\mathrm{c}}(v=1)$.

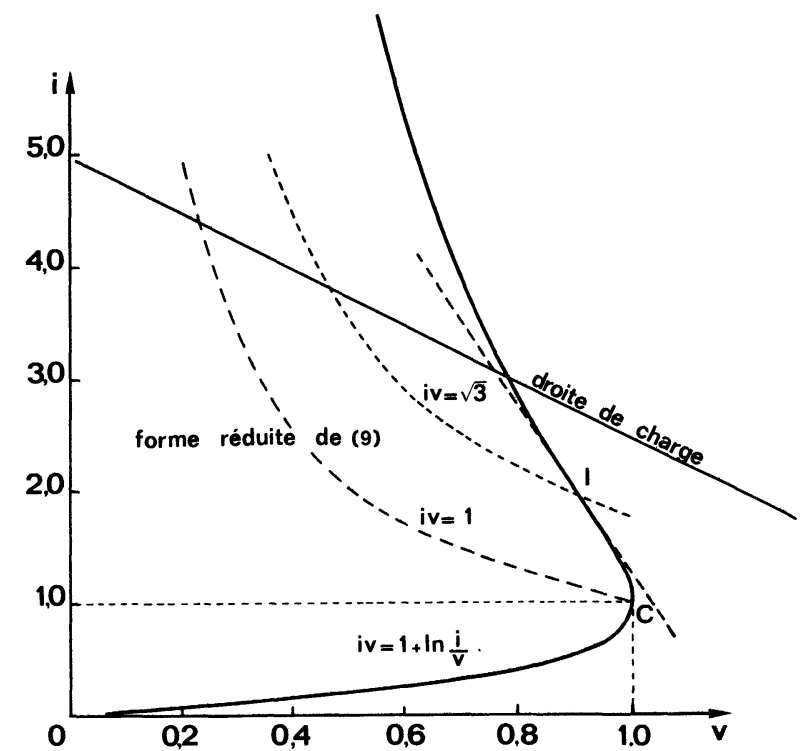

Fig. 2. - Caractéristique courant réduit-tension réduite pour un échantillon isotherme. Le point critique $v=i=1$ correspond au claquage thermique. On a indiqué sur la figure l'hyperbole critique $(i v=1)$, l'hyperbole passant par le point d'inflexion de la caractéristique $(i v=\sqrt{3}$ ) et une droite de charge coupant la caractéristique en un point dans la zone de pente négative.

[Reduced current-reduced voltage for an isothermal sample. The critical point $v=i=1$ corresponds to thermal breakdown. We have drawn the critical hyperbola $(i v=1)$, the hyperbola joining the inflexions of the characteristics $(i v=\sqrt{3})$ and a load line intersecting the characteristics at a point in the negative slope range.] 
Pour obtenir expérimentalement toute la caractéristique y compris sa branche à pente négative, il faut utiliser un générateur de courant, par exemple une source de tension $V_{\mathrm{G}}$ dont le débit est limité par une résistance $R$ en série avec l'échantillon. La tension $V$ aux bornes de l'échantillon est alors :

$$
V=V_{\mathrm{G}}-S I R
$$

où $S$ est la surface de l'échantillon, et la valeur absolue $1 / S R$ de la pente de la droite de charge $I=\left(V_{\mathrm{G}}-V\right) / S R$ doit être inférieure au module $|\mathrm{d} I / \mathrm{d} V|_{\text {inf }}$ de la pente de la caractéristique au point d'inflexion. Ceci s'exprime par l'inégalité :

$$
S R>\left|\frac{\mathrm{d} V}{\mathrm{~d} I}\right|_{\text {inf }} .
$$

Le point d'inflexion, tel que $\frac{\mathrm{d}^{2} i}{\mathrm{~d} v^{2}}=0$, correspond à $i v=\sqrt{3}$, soit

$$
(I V)_{\mathrm{inf}}=2 \sqrt{3} \Gamma \theta .
$$

D'autre part, d'après la relation (14),

$$
\left(\frac{V}{I}\right)_{\text {inf }}=\frac{L}{\sigma_{0}} \mathrm{e}^{-\sqrt{3}}=S R_{0} \mathrm{e}^{-\sqrt{3}}
$$

où $R_{0}$ est la résistance de l'échantillon à la température ambiante.

En combinant (17), (19) et (20), on obtient :

$$
\left|\frac{\mathrm{d} V}{\mathrm{~d} I}\right|_{\text {inf }}=\mathrm{e}^{-\sqrt{3}} \frac{\sqrt{3}-1}{\sqrt{3}+1} S R_{0}=0,0474 S R_{0}
$$

et la condition nécessaire (inégalité (18)) pour qu'on puisse obtenir toute la caractéristique se réduit à :

$$
R>0,0474 R_{0} .
$$

3.2 ÉCHANTILLON NON OHMIQUE. - Si maintenant on tient compte de la dépendance de $\sigma$ avec le champ $E$, donnée par la relation (4), les équations (8) et (9) restent inchangées. On a encore

$$
\begin{aligned}
T_{\mathrm{c}} & =T_{0}+\theta \\
W_{\mathrm{c}} & =I_{n \mathrm{c}} V_{n \mathrm{c}}=2 \Gamma \theta
\end{aligned}
$$

où $I_{n c}$ et $V_{n c}$ sont les nouveaux courant et tension critiques.
Pour obtenir l'équation de la caractéristique et les nouvelles valeurs critiques $V_{\mathrm{c}}, E_{\mathrm{c}}$ et $I_{\mathrm{c}}$, il faut faire une hypothèse sur la forme de la fonction $f(E)$. La forme la plus simple qu'on puisse donner à cette fonction croissante de $E$ est :

$$
f(E) \propto E^{n}
$$

avec $n>0$.

Avec une telle fonction, la densité de courant $I$ devient :

$$
I=\sigma_{0} \exp \left(\frac{T-T_{0}}{\theta}\right) E^{n+1} .
$$

En tenant compte de (6), l'équation intrinsèque de la caractéristique s'écrit :

$$
I V=2 \Gamma \theta \ln \left(\frac{I L^{n+1}}{\sigma_{0} V^{n+1}}\right)
$$

et peut se mettre sous la forme réduite

$$
i_{n} v_{n}=1+\ln \left\{i_{n} v_{n}^{-(n+1)}\right\}
$$

avec $i_{n}=I / I_{n c}$ et $v_{n}=V / V_{n c}$. Les nouvelles quantités critiques sont données dans le tableau II.

4. Traitement tenant compte du profil de température dans l'échantillon. - Dans cette section, nous allons encore admettre qu'il existe une discontinuité de température aux interfaces, mais nous tenons compte cette fois du profil de température à l'intérieur du matériau, représenté schématiquement sur la figure 3 en fonction de l'ordonnée $z$.

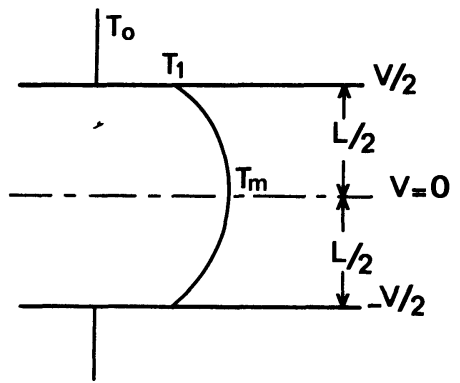

Fig. 3. - Profil de température à l'intérieur d'un échantillon homogène non isotherme, avec transfert convectif aux électrodes.

[Temperature profile across a non isothermal homogeneous sample with convective transfer at the electrodes.]

Tableau II. - Les conditions critiques pour un échantillon obéissant à la relation (4) avec $f(E) \propto E^{n}$.

[The critical conditions for a sample obeying eq. (4) with $f(E) \propto E^{n}$.]

Quantités critiques

Température critique $T_{n \mathrm{c}}$

Puissance critique $W_{n c}$

Tension critique $V_{n c}$

Champ critique $E_{n c}$

Courant critique $I_{n c}$
Formules

$$
\begin{gathered}
T_{0}+\theta \\
2 \Gamma \theta
\end{gathered}
$$

$L\left(2 \Gamma \theta / \mathrm{e} \sigma_{0} L\right)^{1 /(n+2)}$

$\left(2 \Gamma \theta / \mathrm{e} \sigma_{0} L\right)^{1 /(n+2)}$

$2 \Gamma \theta / L V_{n c}$
Remarques

$\forall n$

$\forall n$

varie en $L^{-1 /(n+2)}$ 
Les équations de continuité du courant et du flux de chaleur dans le matériau sont respectivement :

$$
I=\sigma(T) E(z)=\sigma(T) \frac{\mathrm{d} V}{\mathrm{~d} z}
$$

et, en appelant $K$ la conductivité thermique du matériau :

$$
\frac{\mathrm{d}}{\mathrm{d} z}\left(K \frac{\mathrm{d} T}{\mathrm{~d} z}\right)=-\sigma(T) E^{2}(z)=-I \frac{\mathrm{d} V}{\mathrm{~d} z}
$$

Une première intégration de $(30)$ donne :

$$
K \frac{\mathrm{d} T}{\mathrm{~d} z}+I V(z)=0,
$$

la constante d'intégration étant nulle puisque $T$ est maximum, par symétrie $T=T_{\mathrm{m}}$, au plan $z=0$.

En combinant (29) à (31), on obtient :

$$
\frac{2 K}{\sigma} \frac{\mathrm{d} T}{\mathrm{~d} z}=-\frac{\mathrm{d} V^{2}}{\mathrm{~d} z}
$$

$$
\text { d'où } \quad V^{2}(z)=2 K \int_{T}^{T_{\mathrm{m}}} \frac{\mathrm{d} T}{\sigma(T)} \text {. }
$$

En utilisant l'approximation (2), la relation (33) devient :

$$
V^{2}(z)=\frac{2 K}{\sigma_{0}} \int_{T}^{T_{\mathrm{m}}} \exp \left(-\frac{T-T_{0}}{\theta}\right) \mathrm{d} T
$$

soit

$V^{2}(z)=\frac{2 K \theta}{\sigma_{0}}\left[\exp \left(-\frac{T-T_{0}}{\theta}\right)-\exp \left(-\frac{T_{\mathrm{m}}-T_{0}}{\theta}\right)\right]$.

Aux interfaces $\left(V( \pm L / 2)= \pm V / 2\right.$ et $\left.T=T_{1}\right)$ :

$$
\frac{V^{2}}{4}=\frac{2 K \theta}{\sigma_{0}}\left[\exp \left(-\frac{T_{1}-T_{0}}{\theta}\right)-\exp \left(-\frac{T_{\mathrm{m}}-T_{0}}{\theta}\right)\right] .
$$

En tenant compte ici encore de la relation (6), et en posant, pour simplifier l'écriture, $\frac{T_{\mathrm{m}}-T_{1}}{\theta}=t$, (36) devient :

$$
V^{2}=\frac{8 K \theta}{\sigma_{0}} \mathrm{e}^{-I V / 2 \Gamma \theta}\left(1-\mathrm{e}^{-t}\right) .
$$

De même, en combinant (32) et (35), on obtient l'équation différentielle pour $T(z)$ :

$$
\begin{aligned}
- & \frac{K}{I} \frac{\mathrm{d} T}{\mathrm{~d} z}=\left(\frac{2 K \theta}{\sigma_{0}}\right)^{1 / 2} \times \\
& \times\left[\exp \left(-\frac{T-T_{0}}{\theta}\right)-\exp \left(-\frac{T_{\mathrm{m}}-T_{0}}{\theta}\right)\right]^{1 / 2} .
\end{aligned}
$$

En désignant par $u^{2}$ la quantité entre crochets dans (38), cette équation devient :

$$
\frac{\mathrm{d} u}{u^{2}+\exp \left[-\left(T_{\mathrm{m}}-T_{0}\right) / \theta\right]}=\frac{I}{\sqrt{2 K \theta \sigma_{0}}} \mathrm{~d} z .
$$

L'équation (39) peut être exploitée de deux manières. D'une part, elle peut être intégrée par rapport à la variable courante $z$, pour obtenir $T(z)$, donc $V(z)$ et $E(z)$. D'autre part, elle peut être intégrée entre le plan médian de l'échantillon et une des interfaces, pour obtenir la relation liant le courant traversant l'échantillon à la tension appliquée. Nous allons traiter successivement ces deux cas.

4.1 INTÉGRATION DE L'ÉQUATION (39) PAR RAPPORT A LA VARIABLE COURANTE $z$. - L'équation (39) s'intègre en donnant :

$$
\operatorname{Arctg}\left[u \mathrm{e}^{\left(T_{\mathrm{m}}-T_{0}\right) / 2 \theta}\right]=\frac{I \mathrm{e}^{-\left(T_{\mathrm{m}}-T_{0}\right) / 2 \theta}}{\sqrt{2 K \theta \sigma_{0}}} z
$$

(la constante d'intégration est nulle car à $z=0$ correspond $u=0$ ).

La relation (40) se met sous la forme simple

$$
\mathrm{e}^{-\left(T_{\mathrm{m}}-T_{0}\right) / 2 \theta}=\cos (z / a)
$$

avec

$$
a=\frac{\sqrt{2 K \theta \sigma_{0}}}{I} \mathrm{e}^{\left(T_{\mathrm{m}}-T_{0}\right) / 2 \theta} .
$$

On en tire la valeur de $T(z)$ donnée dans le tableau III.

Tableau III. - Profil à l'équilibre de la température $T, d u$ potentiel $V$ et du champ $E$ dans un échantillon ohmique.

[Steady state profile of the temperature $T$, the potential $V$ and the field $E$ through an ohmic sample.]

\section{Quantités}

Température $T(z)$

Potentiel $V(z)$

Champ $E(z)$

(Le logarithme dans la relation (45) étant négatif, $T_{\mathrm{m}}$ est bien la température maximale.)

En vertu de la relation (31), on obtient $V(z)$ par dérivation :

$$
V(z)=\frac{2 K \theta}{I a} \operatorname{tg}(z / a) .
$$

En tenant compte de (42) et de la valeur prise par $V$ aux limites $( \pm L / 2)$ :

$$
V / 2=\sqrt{2 K \theta / \sigma_{0}} \mathrm{e}^{-\left(T_{\mathrm{m}}-T_{0}\right) / 2 \theta} \operatorname{tg}(z / a),
$$

$V(z)$ prend la forme (46) donnée au tableau III. Enfin, une seconde dérivation donne le champ électrique local transcrit dans le même tableau. 
4.2 Caractéristioue courant-tension. - Pour obtenir la caractéristique courant-tension, on peut utiliser l'équation aux limites (44), dans laquelle la différence $\left(T_{\mathrm{m}}-T_{0}\right)$ est décomposée en

$$
\left(T_{\mathrm{m}}-T_{1}+T_{1}-T_{0}\right),
$$

soit, en vertu de (6), $T_{\mathrm{m}}-T_{1}+\frac{I V}{2 \Gamma}$. On a alors :

$$
\begin{aligned}
& V=\sqrt{\frac{8 K \theta}{\sigma_{0}}} \exp \left(-\frac{t}{2}-\frac{I V}{4 \Gamma \theta}\right) \times \\
& \times \operatorname{tg}\left[\frac{I L}{\sqrt{8 K \theta \sigma}} \exp \left(-\frac{t}{2}-\frac{I V}{4 \Gamma \theta}\right)\right] .
\end{aligned}
$$

En comparant les expressions de $V$ données par (37) et (48), on obtient :

$$
\operatorname{tg}\left[\frac{I L}{\sqrt{8 K \theta \sigma_{0}}} \exp \left(-\frac{t}{2}-\frac{I V}{4 \Gamma \theta}\right)\right]=\sqrt{\mathrm{e}^{t}-1} .
$$

En utilisant maintenant les nouvelles variables :

et

$$
\vartheta=V / V_{0} \text { avec } \quad V_{0}=\sqrt{\frac{8 K \theta}{\sigma_{0}}}
$$

$$
\mathfrak{J}=I / I_{0} \quad \text { avec } \quad I_{0}=\sigma_{0} V_{0} / L,
$$

les relations (37) et (49) s'écrivent respectivement, en posant en outre $\gamma=\Gamma L / 4 K$,

$$
\begin{aligned}
\mho^{2} \mathrm{e}^{\jmath \mho / \gamma} & =1-\mathrm{e}^{-t} \\
\operatorname{Arctg} \sqrt{\mathrm{e}^{t}-1} & =J \mathrm{e}^{-\jmath \mho / 2 \gamma} \mathrm{e}^{-t / 2} .
\end{aligned}
$$

Finalement, l'élimination de $\mathrm{e}^{t}$ entre les relations (50) et $\left(50^{\prime}\right)$ conduit, après quelques manipulations algébriques, à la relation :

$$
\sin \left[\jmath \mho \sqrt{\frac{1}{\mathcal{V}^{2} \mathrm{e}^{\jmath \mho / \gamma}}-1}\right]=\mathcal{V} \mathrm{e}^{\jmath \mho / 2 \gamma} .
$$

La relation (51) représente la nouvelle caractéristique réduite, en fonction du paramètre $\gamma$ qui caractérise le rapport entre le transfert convectif aux interfaces et la conductivité thermique du matériau.

Les caractéristiques correspondant à la relation (51), calculées par ordinateur selon la méthode exposée Annexe II, sont représentées sur la figure 4. On notera que, comme pour le cas du matériau isotherme, les caractéristiques s'incurvent vers l'axe des $\mathfrak{J}$, et présentent une branche à résistance négative, lorsque la puissance dissipée dépasse une valeur critique qui dépend, bien entendu, de $\gamma$. Toutefois, il faut noter que la puissance critique réduite augmente considérablement lorsque $\gamma$ devient très grand par rapport à l'unité. La limite $\gamma \rightarrow \infty$ correspond au cas où les électrodes sont des puits de chaleur $\left(T_{1}=T_{0}\right)$, qui est représenté sur la figure 5. Dans ce cas, la rela-

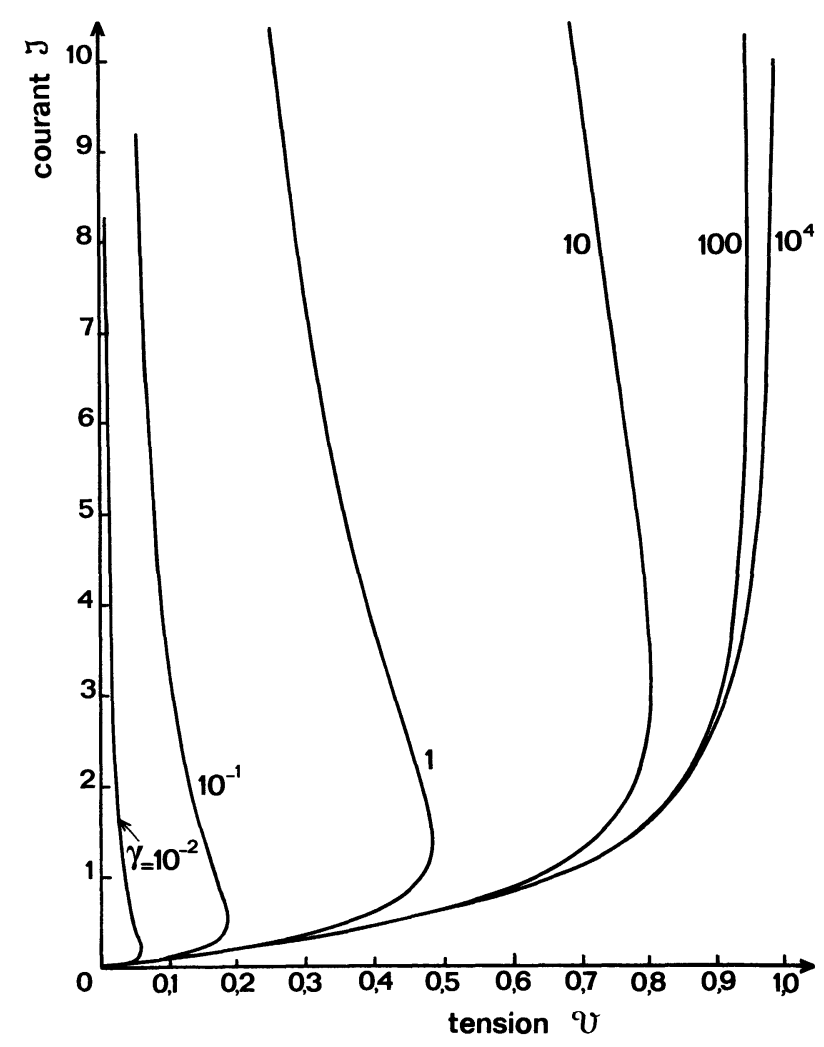

Fig. 4. - Caractéristiques courant réduit-tension réduite dans le cas d'un échantillon non isotherme, pour différentes valeurs du rapport $\gamma=\Gamma L / 4 K$.

[Reduced current-reduced voltage characteristics for a non isothermal sample for various values of the ratio $\gamma=\Gamma L / 4 \mathrm{~K}$.]

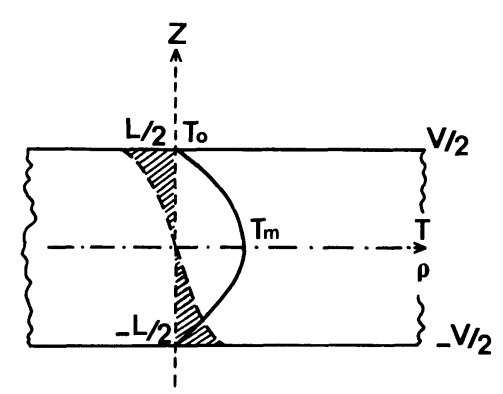

Fig. 5. - Schéma représentant la densité de charge $\rho$ (zone hachurée) résultant de la superposition du champ et du profil de température.

[Schematic representation of the charge density $\rho$ (darkened area) resulting from the superposition of the field and the temperature profile.]

tion (51) se simplifie et le courant réduit se met sous la forme analytique simple :

$$
J=\frac{\operatorname{Arcsin} \vartheta}{\sqrt{1-\vartheta^{2}}} .
$$

Cette caractéristique limite, asymptotique à l'axe $\vartheta=1$, a son point critique rejeté à l'infini sur cet axe, et elle ne présente pas de branche à pente négative. 
Tableau IV. - Valeurs du champ critique limite $E_{0}=\frac{1}{L} V_{0}$ avec $V_{0}=\sqrt{8 K \theta / \sigma_{0}}$, pour différentes valeurs de $L$. [Values of the limit critical field $E_{0}=V_{0} / L$ with $V_{0}=\sqrt{8 K \theta / \sigma_{0}}$ for various.]

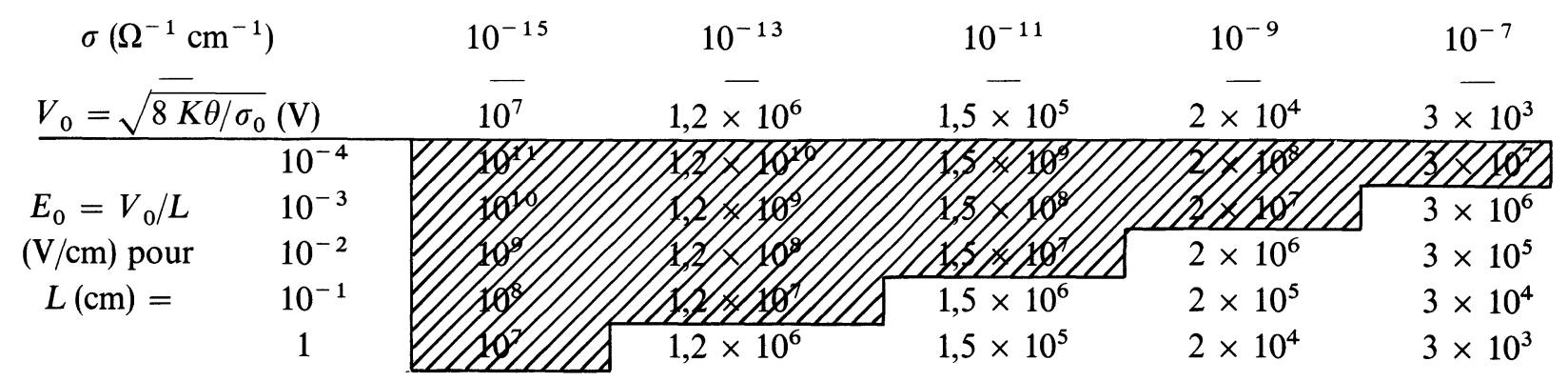

En d'autres termes, la présence sur la caractéristique d'une branche à pente négative est liée à une discontinuité de température aux interfaces, discontinuité qui ne peut exister - en principe - qu'en transfert convectif ou radiatif, mais pas dans un échantillon encapsulé.

Dans les conditions $(\gamma \rightarrow \infty)$ de la relation (52), le claquage thermique se produit lorsque la tension aux bornes de l'échantillon a la valeur $V_{0}=\sqrt{8 \mathrm{~K} \theta / \sigma_{0}}$, indépendante de l'épaisseur.

Dans le tableau IV, on a inscrit le champ limite $E_{0}=V_{0} / L$ pour différentes épaisseurs et différentes valeurs de la conductivité $\sigma_{0}$. Lorsque le champ limite est très supérieur à $10^{6} \mathrm{~V} . \mathrm{cm}^{-1}$ (zone hachurée du tableau) le claquage est de nature électronique, puisque le matériau ne peut pas dissiper assez de chaleur par effet Joule. Dans ce tableau, la conductivité thermique $K$, supposée de l'ordre de $10^{-3} \mathrm{~W} \cdot \mathrm{cm}^{-1} \cdot \mathrm{deg}^{-1}$ pour un matériau très isolant, $\left(\sigma \sim 10^{-15} \Omega^{-1} \mathrm{~cm}^{-1}\right)$ a été augmentée pour les matériaux plus conducteurs, et fixée arbitrairement à $10^{-2} \mathrm{~W} \cdot \mathrm{cm}^{-1} \cdot \mathrm{deg}^{-1}$ pour un matériau relativement conducteur (colonne droite du tableau : $\left.\sigma \sim 10^{-7} \Omega^{-1} \mathrm{~cm}^{-1}\right)$.

Les chiffres du tableau ne sont qu'indicatifs, mais ils montrent clairement qu'un échantillon très mince d'un matériau très isolant ne peut être le siège d'un claquage purement thermique dans les conditions ci-dessus, surtout si cet échantillon est homogène et encapsulé. Un claquage pseudo-thermique peut toutefois se produire si un point chaud apparaît localement à une interface, par exemple par dissipation locale d'une forte densité de courant d'émission. Ce processus a été identifié en phase liquide [4], mais il ne s'agit plus d'un processus dissipatif intrinsèque.

5. Polarisation induite et permittivité. - Nous évoquons pour terminer un aspect peu exploité du modèle. Il s'agit de la polarisation induite dans le matériau du fait des gradients de température qui s'y installent [5]. Pour simplifier, nous traiterons cette polarisation dans le cas particulier $\gamma=\infty$.

$\mathrm{Au}$ gradient de température correspond un gradient de conductivité, mais comme la densité de courant à l'équilibre est uniforme, le champ $E$ se répartit de façon à compenser le gradient de conductivité. En vertu de la loi de Poisson, cette répartition du champ $E$ implique la présence d'une charge d'espace $\rho(z)=\varepsilon \mathrm{d} E / \mathrm{d} z$ ( $\varepsilon$ étant supposé uniforme).

Le moment dipolaire ainsi acquis, à l'équilibre, par unité de surface de l'échantillon est (cf. Annexe III) :

$$
\mu=\varepsilon\left(L I / \sigma_{0}-V\right) .
$$

La polarisation par unité de surface d'échantillon est donc :

$$
P=\frac{\mu}{L}=\varepsilon\left(I / \sigma_{0}-E_{\mathrm{a}}\right)
$$

où $E_{\mathrm{a}}$ est le champ appliqué.

La polarisation calculée ci-dessus se met sous la forme

$$
P=\varepsilon(\mathfrak{J}-\vartheta) V_{0} / L
$$

qui devient, avec la valeur de J donnée par (52) :

$$
P=\varepsilon \frac{V_{0}}{L}\left(\frac{\operatorname{Arcsin} \vartheta}{\sqrt{1-\vartheta^{2}}}-\vartheta\right) .
$$

Si $\mho$ est assez petit devant l'unité (disons $\vartheta \leqslant 0,1$ ), on peut remplacer la parenthèse de (56) par le premier terme de son développement suivant les puissances de $v$.

$$
P=\left(2 \varepsilon V_{0} / 3 L\right) \mho^{2} .
$$

La permittivité statique $\varepsilon_{\mathrm{s}}$ de l'échantillon est définie par l'égalité :

$$
P=\left(\varepsilon_{\mathrm{s}}-\varepsilon\right) V / L
$$

En utilisant (57) et en se souvenant que $V / V_{0}=v$, $\varepsilon_{\mathrm{s}}$ s'écrit :

$$
\varepsilon_{\mathrm{s}} \simeq(1+2 \mho / 3) \varepsilon .
$$

L'effet du terme en $\vartheta$ de la relation ci-dessus peut ne pas être négligeable pour des échantillons assez conducteurs. Par exemple, on voit sur la colonne de droite du tableau III $\left(V_{0}=3000 \mathrm{~V}\right)$ qu'une tension appliquée d'une centaine de volts suffit pour que $\varepsilon_{\mathrm{s}}$ excède $\varepsilon$ de $2,2 \%$. 
Pour estimer la fréquence maximale à laquelle on peut espérer mesurer l'effet du terme en $\mho$ de (59), on peut assimiler cette fréquence à l'inverse de la constante de temps $\tau_{\mathrm{ce}}$ de formation de la charge d'espace, calculée par Von Hippel et al. [6].

Cette constante de temps peut se mettre sous la forme $\tau_{\mathrm{ce}}=\sqrt{\tau \tau_{\mathrm{T}}}$ où $\tau$ est le temps de relaxation diélectrique $\varepsilon / \sigma$ du matériau, et $\tau_{\mathrm{T}}$ le temps de transfert $L^{2} / \mu V$ des porteurs d'une électrode à l'autre en champ uniforme.

La fréquence maximale se situe dans une zone acceptable si le matériau est relativement conducteur et si son épaisseur est assez mince.

6. Conclusion. - Une reformulation du problème du claquage purement dissipatif de matériaux non métalliques homogènes a permis de confirmer que le champ critique décroît toujours quand l'épaisseur de l'échantillon augmente. Toutefois, la forme précise de la relation entre champ critique et épaisseur peut prendre des formes très différentes selon la façon dont la conductivité électrique du matériau dépend du champ et selon la nature du transfert de chaleur aux interfaces.

Pour un échantillon de conductivité thermique élevée $(\gamma \ll 1)$ et de conductivité électrique superohmique du type $\sigma \propto E^{n}$, le champ critique varie en $L^{-1 /(n+2)}$.

$\mathrm{Au}$ contraire, pour un échantillon ohmique dont la conductivité thermique est faible $(\gamma \gg 1)$, le champ critique varie pratiquement en $L^{-1}$.

Les deux cas mentionnés ci-dessus sont bien entendu des cas extrêmes, qui encadrent les comportements réels, mais ils montrent que la variation du champ critique avec l'épaisseur ne suffit pas - à elle seule - pour attribuer au phénomène un mécanisme précis.

Par ailleurs, on a établi que, dans le cadre d'un modèle purement dissipatif unidimensionnel, la présence d'une branche à résistance négative dans la caractéristique courant-tension est liée à une discontinuité de la température aux interfaces.

Enfin, on a montré par le calcul que l'application d'un champ électrique alternatif d'amplitude élevée sur une plaque d'un matériau non métallique relativement conducteur peut provoquer la relaxation d'une charge d'espace d'origine thermique et produire ainsi, avant tout effet disruptif, une augmentation mesurable de la partie réelle de la permittivité basse fréquence, qui pourrait être utilisée comme un signe précurseur de la rupture thermique.

Cet effet, de sens opposé à celui qui correspond à l'orientation de dipôles permanents (saturation de Langevin) n'est pas le seul qui puisse augmenter la permittivité. L'orientation de dipoles induits [7], la création de dipôles par électrodissociation de quadrupôles [8] peut également augmenter $\varepsilon^{\prime}$, mais ces effets ne sont observables qu'en solution. Une augmentation de la permittivité avec le champ électrique a été mise en évidence avec des films très isolants [9], mais cet effet, lié à l'existence de barrières par accumulation de porteurs aux interfaces, est un effet extrinsèque qui sera évoqué dans une autre publication.

\section{ANNEXE I}

On se propose dans cette annexe d'évaluer la précision de l'approximation de Wagner, faite pour $T=T_{0}$, au voisinage du point critique. L'identification des relations d'Arrhénius (1) et de Wagner (2) pour $T=T_{0}$ conduit à :

$$
\sigma_{0}=\sigma_{\infty} \exp \left(-U / k T_{0}\right)
$$

et

$$
1 / \theta=U / k T_{0}^{2} .
$$

De (A.1.1) et (A.1.2), on déduit la relation :

$$
\sigma_{\infty}=\sigma_{0} \exp \left(T_{0} / \theta\right)
$$

donnant $\sigma_{\infty}$ en fonction des paramètres de la relation de Wagner.

La température critique $T_{\mathrm{cA}}$ obtenue à partir de la relation d'Arrhénius résulte des deux équations ci-dessous :

$$
V_{\mathrm{cA}}^{2} \sigma_{\infty} \exp \left(-U / k T_{\mathrm{cA}}\right)=2 \Gamma L\left(T_{\mathrm{cA}}-T_{0}\right)
$$

et

$$
V_{\mathrm{cA}}^{2} \sigma_{\infty}\left(U / k T_{\mathrm{cA}}^{2}\right) \exp \left(-U / k T_{\mathrm{cA}}\right)=2 \Gamma L
$$

qui donnent

$$
k T_{\mathrm{cA}}^{2} / U=T_{\mathrm{cA}}-T_{0} .
$$

En utilisant (A.1.2), (A.1.6) prend la forme :

$$
\left(T_{\mathrm{cA}} / T_{0}\right)^{2}=\left(T_{\mathrm{cA}}-T_{0}\right) / \theta .
$$

La résolution approchée de cette équation, obtenue en développant la racine de son discriminant, donne :

$$
T_{\mathrm{cA}} / T_{0}=1+x+2 x^{2}+\cdots
$$

avec $x=\theta / T_{0}$.

On en tire l'écart relatif $\Delta T / T_{\mathrm{c}}$ :

$$
\Delta T / T_{\mathrm{cW}} \simeq\left(T_{\mathrm{cA}}-T_{\mathrm{cW}}\right) / T_{\mathrm{cW}} \simeq 2 x^{2} .
$$

De même, pour évaluer l'approximation sur $V_{c}$, les relations (A.1.3) et (A.1.4) donnent :

$$
V_{\mathrm{cA}}^{2} \sigma_{0} \mathrm{e}^{x+1}=2 \Gamma L \theta(1+2 x+\cdots) .
$$

En utilisant le développement limité de $\mathrm{e}^{x}$, la relation précédente devient :

$$
V_{\mathrm{cA}}^{2} \quad \sigma_{0} \mathrm{e}=2 \Gamma L \theta(1+x+\cdots)
$$


d'où l'on tire l'écart relatif sur la tension critique :

$$
\Delta V / C_{\mathrm{cW}}=\left(V_{\mathrm{cA}}-V_{\mathrm{cw}}\right) / V_{\mathrm{cw}} \simeq x / 2 .
$$

Enfin, la puissance critique $W_{\mathrm{cA}}$ est :

$$
W_{\mathrm{cA}}=2 \Gamma \theta(1+2 x+\cdots) \simeq W_{\mathrm{cW}}(1+2 x)
$$

de sorte que

$$
\Delta W / W_{\mathrm{cw}} \simeq 2 x .
$$

Le courant critique $I_{\mathrm{cA}}$ est alors donné par :

$$
I_{\mathrm{cA}}=(1+2 x+\cdots) /(1+x / 2+\cdots) I_{\mathrm{cW}}
$$

ce qui donne :

$$
\Delta I / I_{\mathrm{cw}} \simeq 3 x / 2 .
$$

On voit ainsi que l'erreur la plus importante provenant de l'approximation de Wagner est faite sur la valeur du courant critique. Pour les valeurs typiques $U=1 \mathrm{eV}$ et $T=300 \mathrm{~K}, \Delta I / I_{\mathrm{cw}}$ vaut $3,7 \%$.

\section{ANNEXE II}

Traitement de l'équation (51). - Les deux membres de la relation (51) impliquent que $\vartheta \mathrm{e}^{\mathrm{j} \mho / 2 \gamma} \leqslant 1$. On peut alors poser :

$$
\vartheta \mathrm{e}^{\jmath \mho / 2 \gamma}=\sin \varphi .
$$

Ainsi, la racine carrée dans le premier membre de (51) vaut-elle $\operatorname{cotg} \varphi$, de sorte que cette relation est équivalente au système paramétrique :

$$
\begin{aligned}
\vartheta \mathrm{e}^{\jmath \mho / 2 \gamma} & =\sin \varphi \\
\text { วข } & =\varphi \operatorname{tg} \varphi .
\end{aligned}
$$

La construction des caractéristiques implique donc l'organigramme suivant :

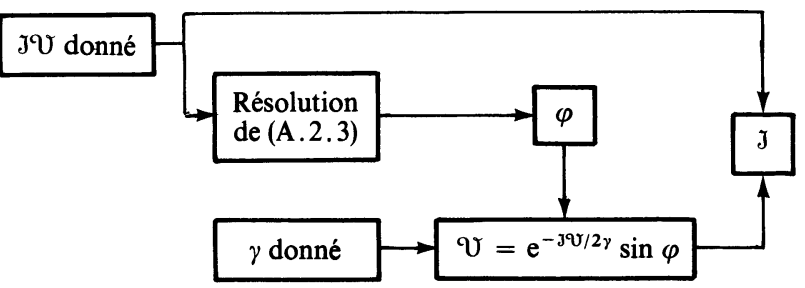

\section{ANNEXE III}

Démonstration de la relation (53). - Par définition, le moment dipolaire électrique est :

$$
\mu=\int_{-L / 2}^{L / 2} z \rho(z) \mathrm{d} z=\varepsilon \int_{-L / 2}^{L / 2} z \mathrm{~d} E
$$

or

$$
\int_{-L / 2}^{L / 2} z \mathrm{~d} E=|z E|_{-L / 2}^{L / 2}-\int_{-L / 2}^{L / 2} E \mathrm{~d} z
$$

donc

$$
\mu=\varepsilon\left[L E(L / 2)-\int_{-L / 2}^{L / 2} E \mathrm{~d} z\right]=\varepsilon[L E(L / 2)-V] .
$$

Comme la température aux interfaces est $T_{0}$, $\sigma( \pm L / 2)=\sigma_{0}$ et $E(L / 2)=I / \sigma_{0}$, et par suite la polarisation par unité de surface de l'échantillon est donnée par la relation (53).

\section{Bibliographie}

[1] Wagner, K. W., Trans. Am. Inst. Electr. Eng. 41 (1922) 288.

[2] Whitehead, S. and Nethercot, W., Proc. Phys. Soc. London 47 (1935) 974.

[3] Neil Berglund, C. and Klein, N., Proc. IEEE 59 (1971) 1099.

[4] Watson, P. K., Annual Report. Conf. on Electrical Insul. NAS-NRC Publ., 1356 (1966) p. 39.

[5] Coelho, R., Agarwal, V. K. and Haug, R., J. Phys. D 10 (1977) 1943.
[6] Von Hippel, A., Phys. Rev. 91 (1953) 568.

[7] Coelho, R., Physics of Dielectrics (Elsevier) 1979, p. 37.

[8] Hellemans, L. and De Maeyer, D., J. Chem. Phys. 63 (1975) 3490.

[9] GoffauX, R., Rev. Gén. Electr. 84 (1975) 249. 